\title{
Vehicle Velocity Control in Case of Two Vehicle Platoon Merging into One Vehicle Platoon
}

\author{
Eisuke Kita and Miichiro Yamada
}

\begin{abstract}
In vehicle platoon, vehicles travel in the row at very short vehicle head distance by the help of the mechanical and the electric systems. The platoon is very important technique for increasing the road traffic capacity safely. The previous studies focus on the safe and stable control of the vehicle platoon. The next aim in the vehicle platoon, however, is the vehicle control of the vehicle platoon in some actual traffic cases. Merging vehicle platoons at the intersection is considered as one of the actual traffic cases in this study. Then, the aim of this study is to discuss the velocity control of the vehicles in the platoon. The velocity control model is defined as the function of the head distance and the velocity difference. The model parameters are determined by stability analysis of the velocity control model. The validity of the model is discussed in the computer simulation and the experiment of LEGO MINDSTORM NXT.
\end{abstract}

Index Terms-Helly model, robot experiment, simulation, vehicle platoon, vehicle following model.

\section{INTRODUCTION}

Vehicle platoon is one of the important attempts to solve the traffic congestion [1]-[3]. In the vehicle platoon, vehicles travel in the row at very small vehicle head distance and as a result, the traffic capacity of the road can be enhanced. The use of the traffic platoon attracts the great interest as the key issue to solve the traffic congestion. A recent study of the vehicle platoon is on the stable platoon of actual vehicles. The interest, in the next study, sifts to the vehicle platoon in some use cases such as the junction, the intersection and so on. The aim of this study is to study the velocity control model of the vehicles in case of merging the vehicle platoons at the junction. At the junction, two platoons merge to one long platoon. In this case, the vehicle velocity should be controlled from the information of one or two nearest vehicles. For this purpose, the velocity control models are defined as the single-leader vehicle and the two-leader vehicles Helly models [4]. The model parameters are determined from the stability analysis of the models. The models are applied for the computer simulation and the experiment of LEGO MINDSTORM NXT. The computer simulation and the experiment results are compared in order to confirm the validity of the models.

The remaining part of this paper is organized as follows. In Section II, the vehicle following models and LEGO

Manuscript received September 23, 2019; revised May 15, 2020. This work was supported by JSPS KAKENHI Grant-in-Aid for Scientific Research (C), Grant Number 15K05760 and 19K04140.

Eisuke Kita is with the Nagoya University, Graduate School of Informatics, Nagoya 464-8601, Japan (e-mail: kita@i.nagoya-u.ac.jp).

Miichiro Yamda is with the Nagoya University, Graduate School of Information Science, Nagoya 464-8601, Japan.
MINDSTORM NXT is introduced briefly as the background of this study. In Section III, the Helly models and stability analysis are performed. The simulation setting is described in Section IV and the computer simulation and experiment results are discussed in Section V. In Section VI, the conclusions are summarized again.

\section{BACKGROUND}

\section{A. Vehicle Following Model}

The velocity control model is defined by means of the vehicle following model.

\section{1) Chandler model}

Chandler model was presented in 1959 [5].

$$
\ddot{x}_{n}(t+T)=a\left(\dot{x}_{n-1}(t)-\dot{x}_{n}(t)\right)
$$

where the variable $\ddot{x}_{n}$ and $\dot{x}_{n}$ denote the acceleration rate and the velocity of the vehicle $n$. The variable $T$ is the delay time of the velocity control. The sensitivity $a$ means the response to the vehicle behavior. Since the vehicle $n-1$ is the nearest forward vehicles of the vehicle $n$, the Chandler model updates the acceleration rate according to the velocity difference $\dot{x}_{n-1}(t)-\dot{x}_{n}(t)$.

\section{2) Newell model}

Newell model was presented in 1961 [6]. In this model, the velocity is defined as the function of the vehicle head distance, which is given as follows.

$$
\dot{x}_{n}(t+T)=G_{n}\left(x_{n-1}(t)-x_{n}(t)\right)
$$

where the notation $G_{n}$ is any nonlinear function of the vehicle head distance.

\section{3) Bierly model}

Bierly model was presented in 1963 [7]. In this model, the acceleration rate is defined as the linear function of the velocity difference and the vehicle head distance, which is given as follows.

$$
\ddot{x}_{n}(t+T)=k_{1}\left(\dot{x}_{n-1}(t)-\dot{x}_{n}(t)\right)+k_{2}\left(x_{n-1}(t)-x_{n}(t)\right)
$$

where the parameter $k_{1}$ and $k_{2}$ are the sensitivity related to the velocity difference and the sensitivity related to the vehicle head distance, respectively.

\section{4) Helly model}

Helly model was presented in 1961 [4]. In this model, the acceleration rate is defined as the linear function of the velocity difference and the vehicle head distance, which is given as follows. 


$$
\begin{gathered}
\ddot{x}_{n}(t+T)=k_{1}\left(\dot{x}_{n-1}(t)-\dot{x}_{n}(t)\right)+k_{2}\left(x_{n-1}(t)-x_{n}(t)-\right. \\
\left.D_{n}(t)\right)
\end{gathered}
$$

where the variable $D_{n}(t)$ denotes the ideal vehicle head distance.

\section{5) Optimal velocity model}

Optimal velocity model was presented in 1995 [8]. In this model, the acceleration rate is given as the function of the difference between the velocity and the optimal velocity function, which is given as follows.

$$
\ddot{x}_{n}(t)=a\left(V\left(\Delta x_{n}\right)-\dot{x}_{n}(t)\right)
$$

where $\Delta x_{n}=x_{n-1}(t)-x_{n}(t)$. The optimal velocity function $V\left(\Delta x_{n}\right)$ is given as follows.

$$
V\left(\Delta x_{n}\right)=\tanh \left(\Delta x_{n}-c\right)+\tanh c
$$

where the parameter $c$ is constant.

\section{B. LEGO MINDSTORMS}

LEGO MINDSTORMS NXT [9] is used in the experiment (Fig.1). In this research, Bricx Command Center (Bricx CC) [10] provided by open source is used as an integrated development environment as a development environment of NXC programming. The programming language is a language developed and distributed as open source Not eXactly C (NXC) [11]. NXC is a language dedicated to LEGO MINDSTORMS developed by John Hansen. The syntax is similar to $\mathrm{C}$, but there are a wealth of instructions to handle MINDSTORMS motors and sensors.

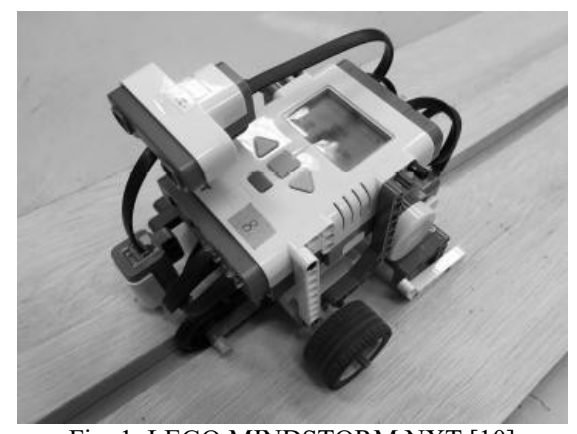

Fig. 1. LEGO MINDSTORM NXT [10].

\section{Vehicle Following Model AND PARAMETER DETERMINATION}

\section{A. Vehicle Following Model}

1) Single-leader vehicle following Helly model

Helly presents the single-leader vehicle following model. The acceleration rate is defined as a function of the velocity difference and the vehicle head distance between the vehicle and its nearest leader vehicle [9], which is defined as

$$
\begin{gathered}
\ddot{x}_{n}(t+T)=\alpha\left(\dot{x}_{n-1}(t)-\dot{x}_{n}(t)\right)+\beta\left[x_{n-1}(t)-x_{n}(t)-\right. \\
\left.D_{n}(t)\right]
\end{gathered}
$$

where the parameters $\alpha$ and $\beta$ are the sensitivities from vehicle $n$ to vehicle $n-1$. The parameter $T$ is the delay time of the control. The function $D_{n}(t)$ denotes the ideal vehicle head distance, which is generally defined as follows.

$$
D_{n}(t)=\gamma_{0}+\gamma_{1} \dot{x}_{n}(t)+\gamma_{2} \ddot{x}_{n}(t)
$$

\section{2) Multi-Leader Vehicles Following Helly Model}

When the original Helly model is extended so as to refer to multiple leader vehicles, the multi-leader vehicles following Helly model is given as

$$
\begin{gathered}
\ddot{x}_{n}(t+T)=\sum_{k=1}^{n}\left\{\alpha_{k}\left(\dot{x}_{n-k}(t)-\dot{x}_{n}(t)\right)+\beta\left[x_{n-k}(t)-\right.\right. \\
\left.\left.x_{n}(t)-D_{n}(t)\right]\right\}
\end{gathered}
$$

where the parameters $\alpha_{k}$ and $\beta_{k}$ are the sensitivities from vehicle $n$ to vehicle $n-k$.

\section{B. Stability Analysis}

\section{1) Single-leader vehicle following Helly model}

Applying Laplace transformation to equation (7) leads to

$$
\begin{aligned}
s e^{T s} V_{n}(s)=\alpha & \left(V_{n-1}(\mathrm{~s})-V_{n}(\mathrm{~s})\right)+\beta\left[\left(\frac{1}{s} V_{n-1}(s)-\frac{1}{s} V_{n}(s)\right)-\right. \\
& \left.\left(\frac{1}{s} \gamma_{0}+\gamma_{1} V_{n}(t)+\gamma_{2} s V_{n}(t)\right)\right]
\end{aligned}
$$

A Pade approximation of the function $e^{T s}$ is given as follows.

$$
e^{T s} \simeq \frac{T^{2} s^{2}+6 T s+12}{T^{2} s^{2}-6 T s+12}
$$

Substituting $\gamma_{0}=0$ and equation (5) into equation (4) leads to

$$
V_{n}(s)=G(s) V_{n-1}(s)
$$

where the transfer function $G(s)$ is given as follows.

$$
G(s)=\frac{\alpha s+\beta}{D(s)}
$$

The characteristic equation $D(s)$ is given as follows.

$$
D(s)=a_{4} s^{4}+a_{3} s^{3}+a_{2} s^{2}+a_{1} s+a_{0}
$$

where

$$
\begin{gathered}
a_{0}=12 \beta \\
a_{1}=12 \alpha-6 T \alpha+12 \beta \gamma_{1} \\
a_{2}=12 \beta \gamma_{2}-6 T \alpha+T^{2} \beta-6 T \beta \gamma_{1}+12 \\
a_{3}=6 T+T^{2} \alpha-6 T \beta \gamma_{2}+T^{2} \beta \gamma_{1} \\
a_{4}=T^{2}+T^{2} \beta \gamma_{2}
\end{gathered}
$$

Applying Hurwitz stability analysis to the characteristic equation (8) leads to the stability region of the sensitivities.

For example, the parameters are specified as $T=0.1, \gamma_{1}=$ 2 and $\gamma_{2}=0$ and then, the stability region of the parameters are shown in Fig. 2.

\section{2) Multi-leader vehicles following Helly model}

Applying Laplace transformation to equation (9) leads to

$$
\begin{gathered}
s e^{T s} V_{n}(s)=\sum_{k=1}^{n}\left\{\alpha_{k}\left(\mathrm{~V}_{n-k}(\mathrm{~s})-\mathrm{V}_{n}(\mathrm{~s})\right)+\right. \\
\beta_{k}\left[\left(\frac{1}{s} V_{n-k}(s)-\frac{1}{s} V_{n}(s)\right)-\left(\frac{1}{s} \gamma_{k 0}+\gamma_{k 1} V_{n}(t)+\right.\right. \\
\left.\left.\left.\gamma k_{2} s V_{n}(t)\right)\right]\right\}
\end{gathered}
$$

Substituting $\gamma_{k 0}=0$ and equation (11) into equation (15) leads to 


$$
V_{n}(s)=G_{n}(s) V_{n-1}(s)
$$

where the transfer function $G_{n}(s)$ is given as follows.

$$
G_{n}(s)=\frac{\sum_{k=1}^{n}\left\{\alpha_{k} s+\beta_{k}\right\}}{D_{n}(s)}
$$

The characteristic equation $D_{n}(s)$ is given as follows.

$$
D(s)=a_{4} s^{4}+a_{3} s^{3}+a_{2} s^{2}+a_{1} s+a_{0}
$$

where

$$
\begin{gathered}
a_{0}=\sum_{k=1}^{n} 12 \beta_{k} \\
a_{1}=\sum_{k=1}^{n}\left\{12 \alpha_{k}-6 T \beta_{k}+12 \beta_{k} \gamma_{k 1}\right\} \\
a_{2}=\sum_{k=1}^{n}\left\{12 \beta_{k} \gamma_{k 2}-6 T \alpha_{k}+T^{2} \beta_{k}-6 T \beta_{k} \gamma_{k 1}\right\}+12 \\
a_{3}=\sum_{k=1}^{n}\left\{T^{2} \alpha_{k}-6 T \beta_{k} \gamma_{k 2}+T^{2} \beta_{k} \gamma_{k 1}\right\}+6 T \\
a_{4}=\sum_{k=1}^{n} T^{2} \beta_{k} \gamma k_{2}+T^{2}
\end{gathered}
$$

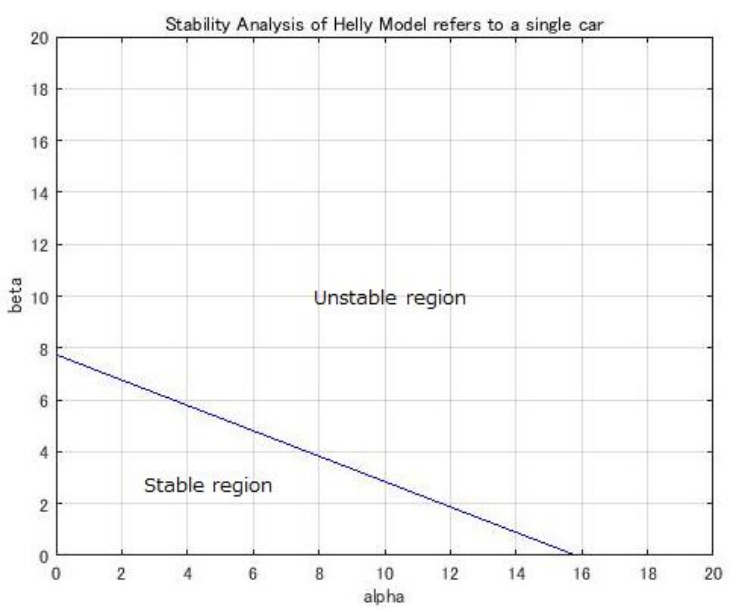

Fig. 2. Stability region of single-leader following Helly model.

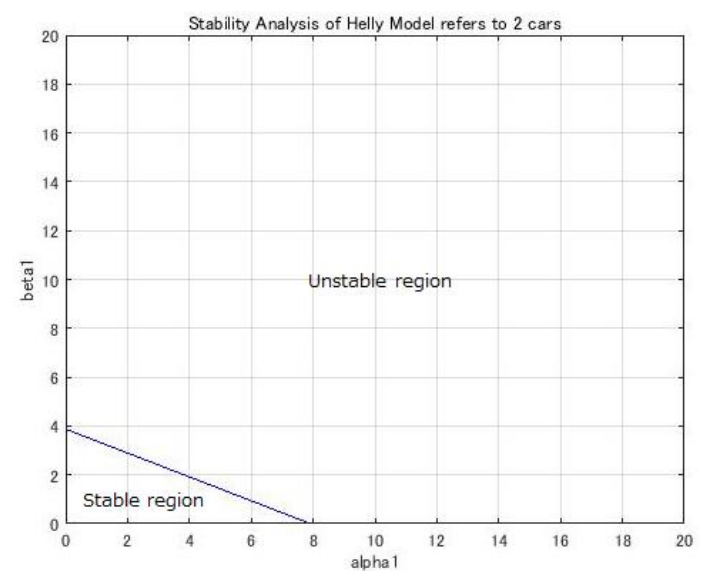

Fig. 3. Stability region of double-leader following Helly model.

Applying Hurwitz stability analysis to the characteristic equation (18) leads to the stability region of the sensitivities.

For example, the parameters are specified as $n=2, T=$ $0.1, \alpha_{1}=\alpha_{2}, \beta_{1}=\beta_{2}, \gamma_{k 1}=2 k$ and $\gamma k_{2}=0$ and then, the stability region of the parameters are shown in Fig.3. It is shown that the stability region in Fig. 3 is smaller than that in Fig. 2.

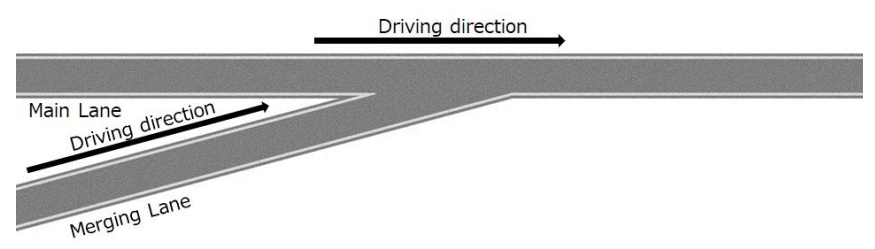

Fig. 4. Road network

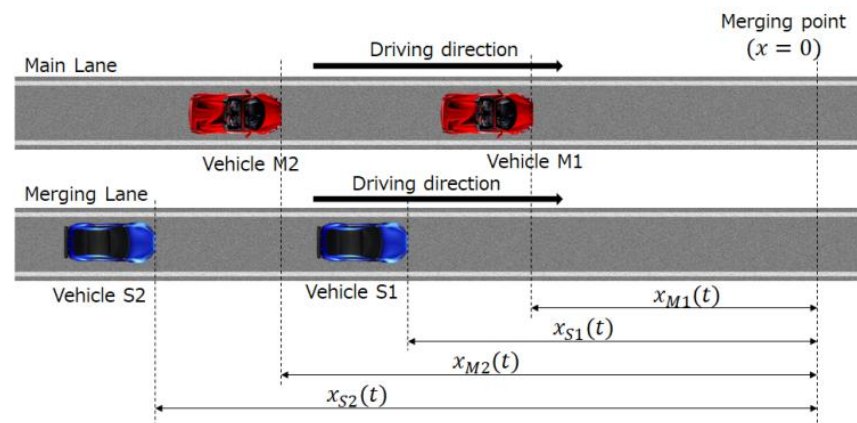

Fig. 5. Initial layout of vehicles.

\section{Simulation Setting}

\section{A. Road Network and Layout of Vehicle}

The road network is composed of a one-lane main road and a one-lane merging road (Fig. 4).

The initial layout of the vehicles is shown in Fig. 5. Two platoons of two vehicles travel on the main road and on the merging road, respectively. After the road junction, two platoons join into single platoon of four vehicles. The vehicles are named as Vehicle M1 and Vehicle M2 on the main road and Vehicle S1 and Vehicle S2 on the merging road, respectively. The position of the vehicle $i$ at the time $t$ is represented with the variable $x_{i}(t)$. The initial positions of the vehicles are given as $x_{M 1}(0)=-200[\mathrm{~cm}], x_{M 2}(0)=-$ $280[\mathrm{~cm}], x_{S 1}(0)=-240[\mathrm{~cm}]$ and $x_{S 2}(0)=-320[\mathrm{~cm}]$.

\section{B. Merging Process of Vehicles}

The successive merging processes of two platoons are illustrated in Fig. 6-Fig. 8.

Before merging, two platoons of two vehicles travel on each road (Fig. 6). The platoon on the main road reaches the junction first. The reference relationship of vehicles is summarized as follows (Fig .9).

(1) M1 travels at a constant velocity.

(2) M2 travels at the velocity which is calculated from equation (1) according to M1.

(3) S1 travels at the velocity which is calculated from equation (1) according to M1.

(4) S2 travels at the velocity which is calculated from equation (2) according to both M2 and S1.

At the merging point, two platoons gather in one platoon (Fig. 7).

After merging, two platoons gather in one platoon (Fig. 8). The reference relationship of vehicles is summarized as follows (Fig. 10).

(1) M1 travels at a constant velocity.

(2) M2 travels at the velocity which is calculated from equation (2) according to both M1 and S1.

(3) S1 travels at the velocity which is calculated from equation (1) according to M1. 
(4) S2 travels at the velocity at the velocity which is calculated from equation (2) according to both M1 and M2.

\section{Sensitivity}

The sensitivity needs to be selected so that the acceleration calculated by each following model does not exceed 77.1 $\left[\mathrm{cm} / \mathrm{s}^{2}\right]$, which is the acceleration limit of the actual machine. The sensitivities in each case are shown in Table I and Table II.

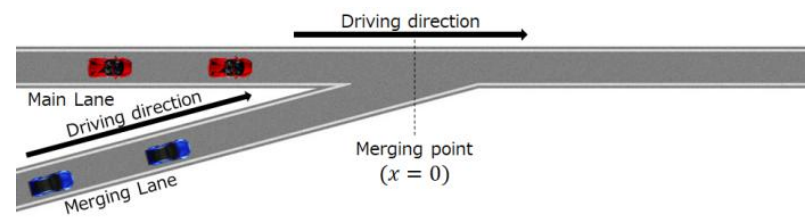

Fig. 6. Before merging point.

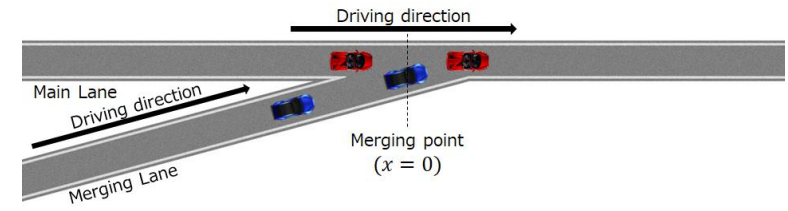

Fig. 7. At merging point.

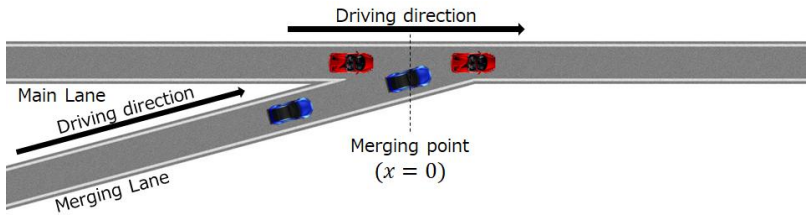

Fig. 8. After merging point.

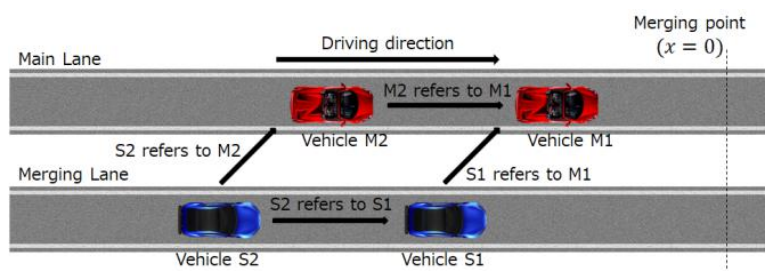

Fig. 9. Reference relationship of vehicles before merging.

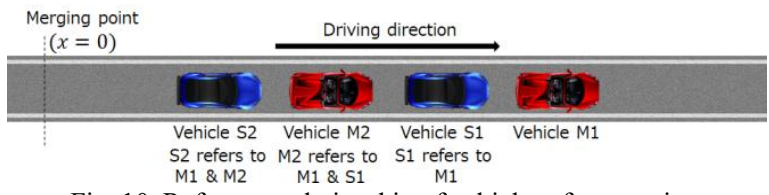

Fig. 10. Reference relationship of vehicles after merging.

TABLE I: SINGLE-LEADER VEHICLE FOLLOWING HELLY MODEL
\begin{tabular}{|c|c|c|c|c|c|}
\hline$\alpha+\beta$ & $\alpha$ & $\beta$ & $\gamma_{0}$ & $\gamma_{1}$ & $\gamma_{2}$ \\
\hline 0.5 & 0.25 & 0.25 & 0 & 1 & 0 \\
\hline 1.0 & 0.5 & 0.5 & 0 & 1 & 0 \\
\hline
\end{tabular}

TABLE II: MUlTI-LEADER VeHICLE FOLlOWING HELly MODEL

\begin{tabular}{|c|c|c|c|c|c|}
\hline$\sum_{k=1}^{2}\left(\alpha_{k}+\beta_{k}\right)$ & $\alpha_{k}$ & $\beta_{k}$ & $\gamma_{k 0}$ & $\gamma_{k 1}$ & $\gamma_{k 2}$ \\
\hline 0.5 & 0.125 & 0.125 & 0 & $2 k$ & 0 \\
\hline 1.0 & 0.25 & 0.25 & 0 & $2 k$ & 0 \\
\hline
\end{tabular}

\section{EXPERIMENT RESULT}

Each vehicle is given as an initial position, and the movement distance is calculated based on the value of the motor's rotation sensor. With the junction as the origin, the current position of the vehicle is estimated. Vehicle distance to the other vehicle ahead is estimated by the infrared sensor. The other vehicle velocity is obtained via bluetooth communication between vehicles. When a cars traveling on a merging lane refers to a car traveling on a main line, the vehicle head distance is calculated by the difference between the positions of each vehicle viewed from the merging point. The control cycle in the experiment is about 0.12 to 0.14 [s]. The total lengths of the main road and the merging road are $700 \mathrm{~cm}$ and $350 \mathrm{~cm}$, respectively.

The course is made with wooden boards, and NXT travels on the road with the grooves between the boards as a guide. Velocity and acceleration of each vehicle are simulated and then, the experimental results of vehicle M1 are compared with the computer simulation results.

When the sum of sensitivities $\sum_{k=1}^{2}\left(\alpha_{k}+\beta_{k}\right)$ is equal to 0.5 and 1.0, the simulation results are shown in Fig. 11 and Fig. 12, respectively. Figures are plotted with time as the horizontal axis and position $[\mathrm{cm}]$, velocity $\left[\mathrm{cm} / \mathrm{s}^{2}\right]$ and acceleration $\left[\mathrm{cm} / \mathrm{s}^{2}\right]$ as the vertical axis, respectively. A blue, a red, a green and a purple lines denote the results of vehicle $\mathrm{M} 1, \mathrm{M} 2, \mathrm{~S} 1$ and S2, respectively. The position in case of $\sum_{k=1}^{2}\left(\alpha_{k}+\beta_{k}\right)=1.0$ is similar to that in case of $\sum_{k=1}^{2}\left(\alpha_{k}+\right.$ $\left.\beta_{k}\right)=0.5$. The velocity recovery is defined as the reduction to $15 \mathrm{~m} / \mathrm{s}$ from the velocity increase. The comparison of the velocity fluctuations in Fig. 11 and Fig. 12 shows that the velocity recovery time in Fig. 12 is shorter than that in Fig. 11. This is because the maximum acceleration rate in $\sum_{k=1}^{2}\left(\alpha_{k}+\right.$ $\left.\beta_{k}\right)=0.5$ is smaller than that in $\sum_{k=1}^{2}\left(\alpha_{k}+\beta_{k}\right)=1.0$.

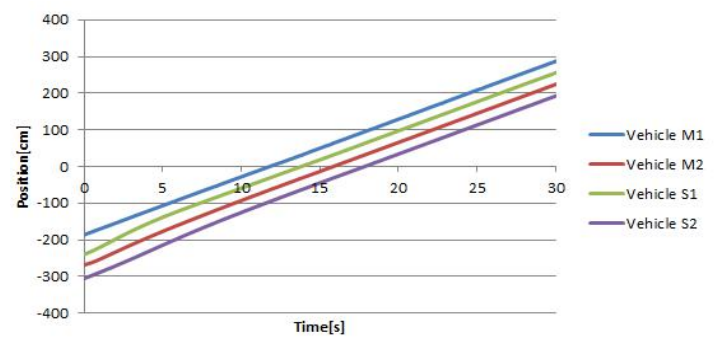

(a) Position

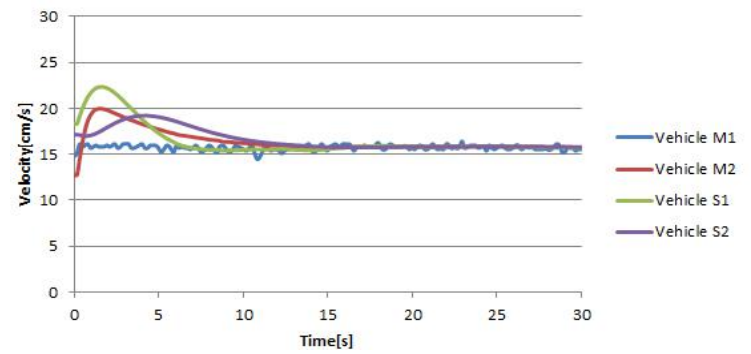

(b) Velocity

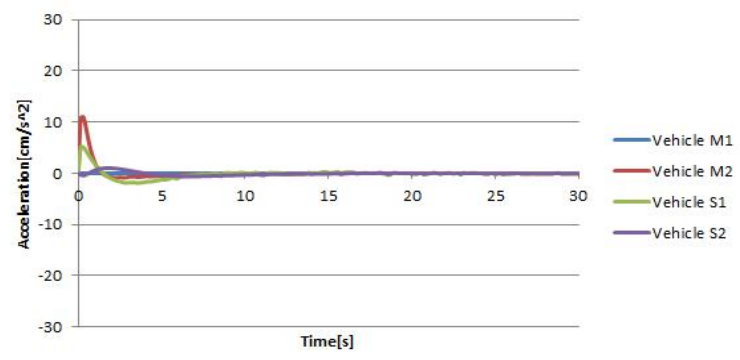

(c) Acceleration

Fig. 11. Computer simulation results in case of $\sum_{k=1}^{2}\left(\alpha_{k}+\beta_{k}\right)=0.5$.

Next, the validity of the model is discussed in the experiments of LEGO MINDSOTRM NXT. The experiment view is shown in Fig. 13. 
The experiment results are shown in Fig. 14 and Fig. 15. The vertical axis of each figure represents the position $[\mathrm{cm}]$, the velocity $[\mathrm{cm} / \mathrm{s}]$ and the acceleration $\left[\mathrm{cm} / \mathrm{s}^{2}\right]$ of each vehicle, and the horizontal axis represents time [s]. A blue, a red, a green and a purple lines denote the results of vehicle M1, M2, S1 and S2, respectively. The large fluctuations in velocity and acceleration are observed in Fig. 14 and Fig. 15. The noise in the velocity and acceleration rate arises from the friction between the vehicle and the experiment course, the measurement error of the ultrasonic sensor and so on.

The mean square error (RMSE) between the experiment and the simulation results are estimated from time $0[\mathrm{~s}]$ to time 30.0 [s], which are hown in Table III. RMSE is calculated by

$$
R M S E=\sqrt{ } \sum_{k=1}^{n}\left(x_{k}-y_{k}\right)^{2} / n
$$

where $x_{k}$ is the measured value, and $y_{k}$ is the calculated value based on the measured value.
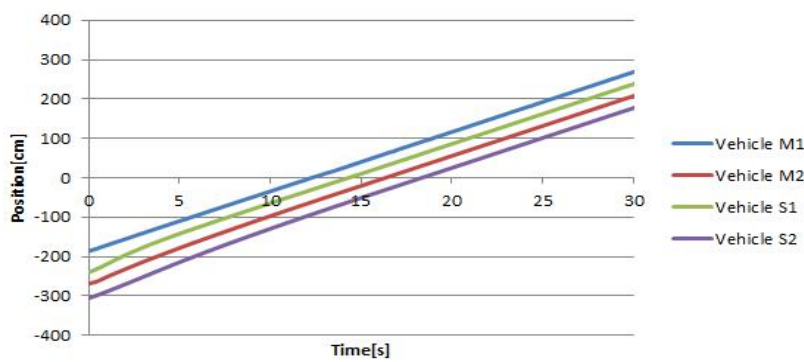

(a) Position
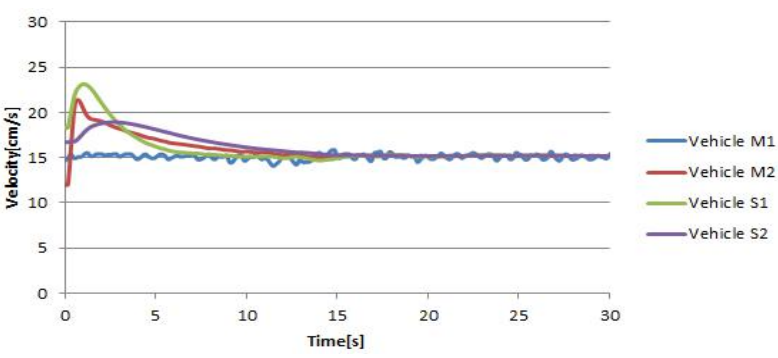

(b) Velocity

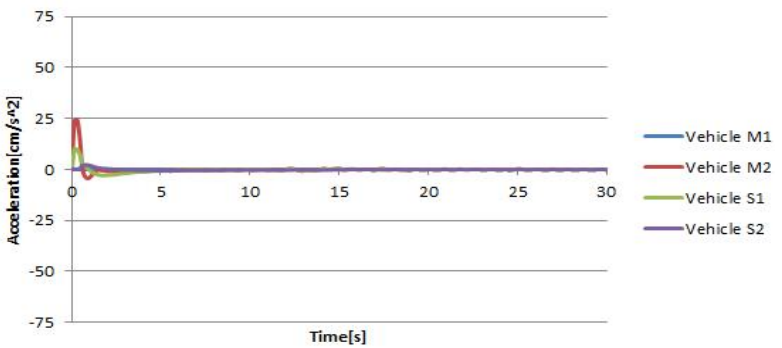

(c) Acceleration

Fig. 12. Computer simulation results in case of $\sum_{k=1}^{2}\left(\alpha_{k}+\beta_{k}\right)=1.0$.

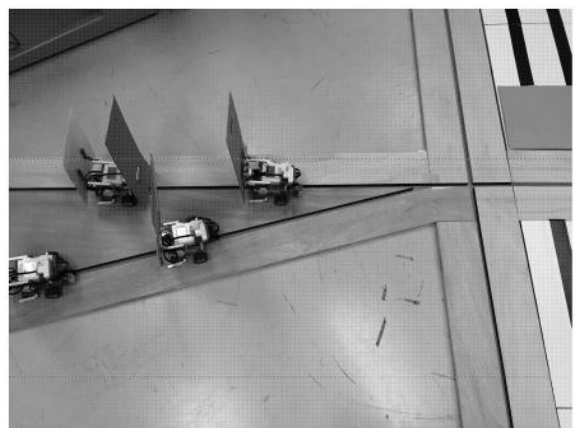

Fig. 13. Experiment view of LEGO MINDSTORM NXTs.
The RMSE of acceleration when the sum of sensitivities is 0.5 is smaller than when it is 1.0 , but there is no significant difference in RMSE of velocity and position.

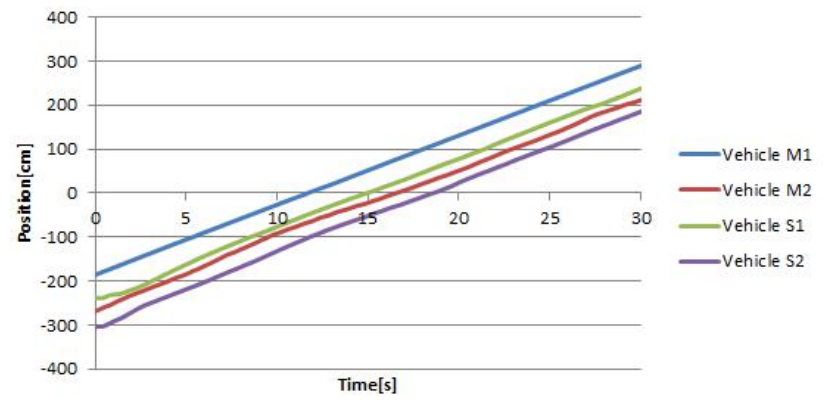

(a) Position

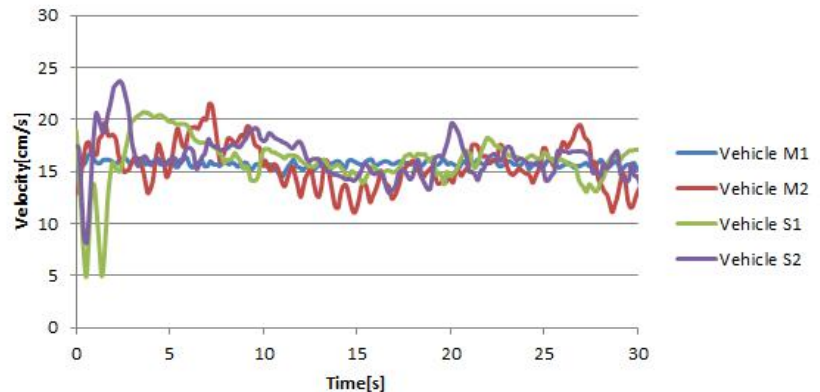

(b) Velocity

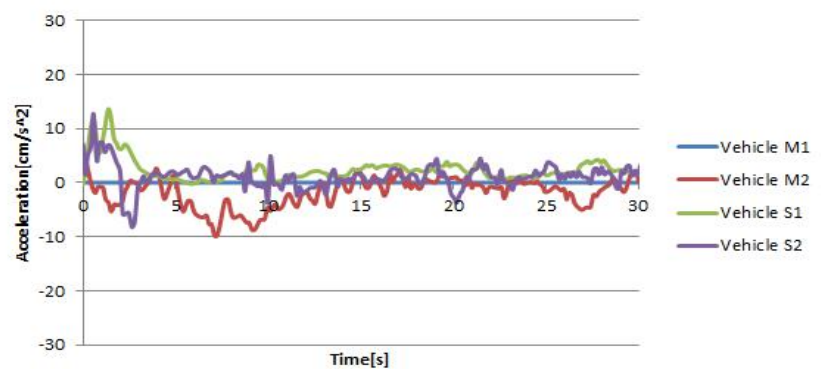

(c) Acceleration

Figure 14: Experimental results in case of $\sum_{k=1}^{2}\left(\alpha_{k}+\beta_{k}\right)=0.5$.

When comparing the vehicles, the RMSE of the vehicle S1 is two times larger than them of the vehicle M2 and S2. This is because the acceleration of LEGO MINDSTORM NXT in the experiment is smaller than the theoretical acceleration of the computer simulation.

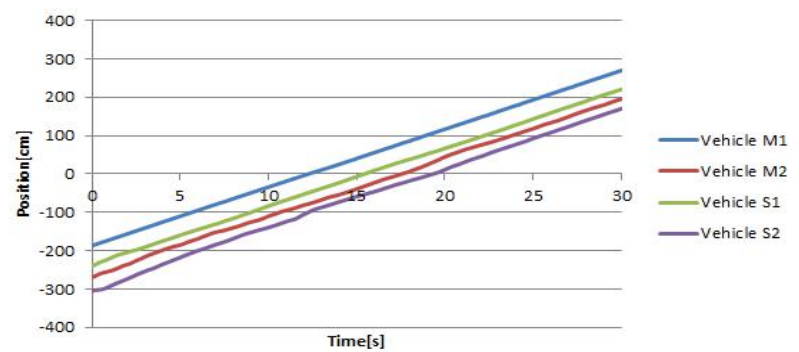

(a) Position

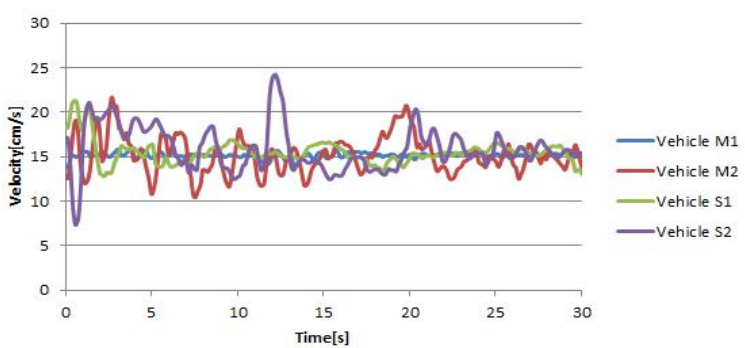

(b) Velocity 


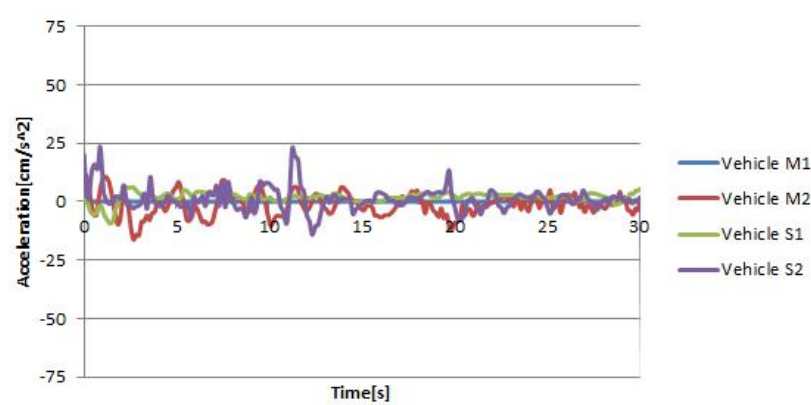

(c) Acceleration

Figure 15: Experimental results in case of $\sum_{k=1}^{2}\left(\alpha_{k}+\beta_{k}\right)=1.0$.

TABLE III: COMPARISON OF EXPERIMENTAL AND SIMULATION RESULTS (a) $\sum_{k=1}^{2}\left(\alpha_{k}+\beta_{k}\right)=0.5$

\begin{tabular}{|c|c|c|c|c|}
\hline Vehicle & M1 & M2 & S1 & S2 \\
\hline Position & 0 & 10.33 & 20.14 & 8.68 \\
\hline Velocity & 0 & 2.22 & 3.09 & 2.01 \\
\hline Acceleration & 0 & 3.34 & 3.18 & 2.63 \\
\hline
\end{tabular}

(b) $\sum_{k=1}^{2}\left(\alpha_{k}+\beta_{k}\right)=1.0$

\begin{tabular}{|c|c|c|c|c|}
\hline Vehicle & M1 & M2 & S1 & S2 \\
\hline Position & 0 & 12.75 & 17.69 & 8.41 \\
\hline Velocity & 0 & 2.36 & 1.72 & 2.37 \\
\hline Acceleration & 0 & 5.33 & 3.21 & 5.22 \\
\hline
\end{tabular}

\section{CONCLUSION}

The vehicle platoon is very important technique for increasing the traffic capacity of road network safely. The previous studies focus on the safe and stable control of the vehicle platoon. The next study in the vehicle platoon is the vehicle platoon in some actual traffic cases. Merging vehicle platoons at the intersection is one of the actual traffic cases. The aim of this study is to discuss the velocity control of the vehicles in the platoon. The velocity control model is defined by means of a Helly model and its extension to two-leader vehicles following Helly model. The model is applied for the computer simulation and the experiment using LEGO MINDSTORMS NXT. The difference between the simulation and the experiment is estimated by the mean square error in two cases that the summation of sensitivities is 0.5 or 1.0. Finally, it is conclude that 0.5 is better than 1.0 for the summation of the sensitivities.

\section{CONFLICT OF INTEREST}

The authors declare no conflict of interest.

\section{AUTHOR CONTRIBUTIONS}

Prof. E. Kita planed this project and wrote the paper. MR. M. Yamada performed the simulation and the experiment.

\section{ACKNOWLEDGMENT}

This work was supported by JSPS KAKENHI Grant-in-Aid for Scientific Research (C), Grant Number $15 \mathrm{~K} 05760$ and 19K04140.

\section{REFERENCES}

[1] C. Bergenhem, S. Shladover, E. Coelingh, C. Englund, and S. Tsugawa, Overview of Platooning Systems, 2012.

[2] M. EL-Zaher, B. Dafflon, F. Gechter, and J.-M. Contet, "Vehicle platoon control with multi-configuration ability," Procedia Computer Science, vol. 9, pp. 1503-1512, 2012.

[3] S. Maiti, S. Winter, and L. Kulik. "A conceptualization of vehicle platoons and platoon operations," Transportation Research Part C: Emerging Technologies, vol. 80, pp. 1-19, 2017.

[4] W. Helly, "Simulation of bottlenecks in single lane traffic flow," in Proc. Theory of Traffic Flow Symposium, 1961, pp. 207-238.

[5] R. E. Chandler, R. Herman, and E. W. Montroll, "Traffic dynamics; studies in car-following," Operations Research, vol. 6, no. 2, pp. 165-184, 1958.

[6] G. F. Newell, "Nonlinear effects in the dynamics of car following," Operations Research.

[7] R. L. Bierley, "Investigation of an inter vehicle spacing display," Highway Research, record 25, pp. 58-75, 1963.

[8] M. Bando, K. Hasebe, K. Nakanishi, A. Nakayama, A. Shibata, and Y. Sugiyama, "Phenomenological study of dynamical model of traffic flow," Journal of Physics I France, vol. 5, pp. 1389-1399, 1995.

[9] LEGO. Lego Mindstorms Education. [Online]. Available: http://www.legoeducation.jp/ mindstorms/

[10] Bricx command center 3.3. [Online]. Available: http://bricxcc.sourceforge.net/

[11] NBC, NXC, SPC. [Online]. Available: $\mathrm{http}: / /$ bricxcc.sourceforge.net/nbc/

Copyright $(\subset 2020$ by the authors. This is an open access article distributed under the Creative Commons Attribution License which permits unrestricted use, distribution, and reproduction in any medium, provided the original work is properly cited (CC BY 4.0).

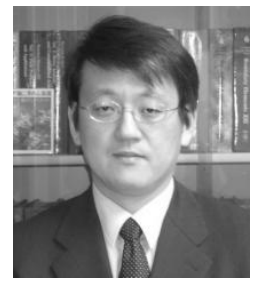

Eisuke Kita is a professor of Graduate School of Informatics in Nagoya University. He received his doctor of engineering degree in Graduate School of Engineering of Nagoya University in 1991. His primary researches are traffic flow control, artificial intelligence and its application for prediction and classification to resolve practical issues. His other research interests also focus on traffic control, machine learning, data mining and evolutionary

computation.

Miichiro Yamada is a master course student of Graduate School of Information Science in Nagoya University. He received his master of information science in Graduate School of Information Science of Nagoya University in 2015. His primary researches are traffic flow control. 